\title{
LIGADURA DE DUCTO BILIAR COMO MODELO DE ESTUDO DA SÍNDROME HEPATOPULMONAR E ESTRESSE OXIDATIVO
}

\author{
Juliana TIEPPO ${ }^{1}$, Rafael VERCELINO ${ }^{1}$, Alexandre Simões DIAS ${ }^{1,2}$, \\ Cláudio Augusto MARRONI ${ }^{3}$ e Norma MARRONI ${ }^{1,4}$
}

RESUMO - Racional - A síndrome hepatopulmonar é caracterizada por uma disfunção hepática e pela existência de dilatações dos vasos pulmonares, levando a alterações nas trocas gasosas, tendo algumas das suas características observadas de forma experimental no modelo de ligadura de ducto biliar. Objetivos - Avaliar o estresse oxidativo no tecido pulmonar de ratos cirróticos por ligadura de ducto biliar comum. Materiais e Métodos - Foram utilizados 12 ratos machos Wistar, pesando entre 200 e 300 g, divididos em dois grupos: controles $(\mathrm{Co}=6)$ e cirróticos $(\mathrm{Ci}=6)$. Foram realizadas avaliações de transaminases, gasometria arterial, avaliação da lipoperoxidação (substâncias reativas ao ácido tiobarbitúrico e quimiluminescência) e quantificação da atividade enzimática antioxidante através das concentrações da enzima superóxido dismutase. Os tecidos analisados para avaliação da síndrome hepatopulmonar foram o fígado cirrótico e o pulmão. Resultados - Os animais com ligadura de ducto biliar apresentaram alteração nas transaminases: aspartato aminotransferase, $\mathrm{Co}=105,3 \pm 43 / \mathrm{Ci}=500,5$ $\pm 90,3$ alanina aminotransferase, $\mathrm{Co}=78,75 \pm 37,7 / \mathrm{Ci}=162,75 \pm 35,4$ e fosfatase alcalina, $\mathrm{Co}=160 \pm 20,45 / \mathrm{Ci}=373,25$ $\pm 45,44$. Em relação à lipoperoxidação e à resposta antioxidante, estas também apresentaram diferenças estatisticamente significativas quando avaliadas no pulmão (substâncias reativas ao ácido tiobarbitúrico) $\mathrm{Co}=0,87 \pm 0,3 / \mathrm{Ci}=2,01 \pm 0,9$; quimiluminescência $\mathrm{Co}=16008,41 \pm 1171,45 / \mathrm{Ci}=20250,36 \pm 827,82 ;$ superóxido dismutase $\mathrm{Co}=6,66 \pm 1,34 / \mathrm{Ci}=16,06 \pm$ 2,67. Conclusĩes - Os dados obtidos sugerem que no modelo experimental de cirrose por ligadura de ducto biliar há aumento significativo da lipoperoxidação no tecido pulmonar, bem como aumento na atividade da enzima antioxidante superóxido dismutase, sugerindo a presença de dano pulmonar decorrente da cirrose biliar secundária.

DESCRITORES - Estresse oxidativo. Ligadura de ducto biliar. Síndrome hepatopulmonar.

\section{INTRODUÇÃO}

A cirrose hepática é uma doença crônica e progressiva, que apresenta um estágio de disfunção hepática lento e irreversível, caracterizando-se pela formação de nódulos hepáticos em conseqüência da fibrose. Estas alterações estruturais constituem as principais respostas do tecido hepático às inúmeras agressões de natureza inflamatória, tóxica, metabólica ou congestiva ${ }^{(22)}$.

Os cirróticos podem apresentar doenças pulmonares comuns, como a bronquite crônica (BC), com relativa freqüência, especialmente em alcoolistas e tabagistas com episódios recurrentes de infecção pulmonar e eventual interferência na função pulmonar ${ }^{(8)}$. A ascite e o derrame pleural podem diminuir a ventilação pulmonar e prejudicar as trocas gasosas.

Nas cirroses, conforme a causa, podem ocorrer situações específicas em que há comprometimento pulmonar e disfunção respiratória, como a alveolite fibrosante e o enfisema pulmonar ${ }^{(3)}$.

A doença hepática crônica pode alterar a vasculatura pulmonar, possibilitando a ocorrência de hipertensão pulmonar, shunts intrapulmonares e a síndrome hepatopulmonar ${ }^{(3)}$. ${ }^{1}$ Laboratório de Fisiologia Digestiva e Estresse Oxidativo, Universidade Federal do Rio Grande do Sul; ${ }^{2}$ Centro Universitário-IPA, Curso de Fisioterapia; ${ }^{3}$ Fundação Faculdade
Federal de Ciências Médicas de Porto Alegre; ${ }^{4}$ Universidade Luterana do Brasil, Porto Alegre, RS.

Endereço para correspondência: Dr. Cláudio Augusto Marroni - Rua José Kanan Aranha, 102 - Bairro Jardim Isabel - 91760-470 - Porto Alegre, RS. E-mail: nmarroni@terra.com.br 
A síndrome hepatopulmonar (SHP) caracteriza-se por uma tríade clínica que inclui doença hepática, anormalidades em trocas gasosas e a presença de dilatações vasculares pulmonares ${ }^{(9)}$.

A obstrução prolongada do ducto biliar é um modelo experimental de cirrose biliar secundária muito utilizado que provoca marcada proliferação ductal, com edema e reações inflamatórias agudas, podendo, ainda, ocasionar alterações sistêmicas ${ }^{(24)}$.

CHANG e O'HARA ${ }^{(5)}$, demonstraram que animais submetidos a ligadura de ducto biliar apresentavam desenvolvimento progressivo de anormalidades nas trocas gasosas, similares àquelas encontradas em humanos com SHP. Observou-se que esses animais cirróticos apresentavam um gradiente alvéoloarterial de oxigênio superior à $100 \%$, associado à hipoxemia moderada (valores inferiores à $85 \mathrm{~mm} \mathrm{Hg}$ ). Estes achados estavam associados à vasodilatação intrapulmonar, atribuída aos elevados valores de oxído nitrico sintase endotelial (eNOS) encontrados no homogeneizado pulmonar ${ }^{(10)}$.

Diversos autores têm demonstrado, que animais com cirrose induzida experimentalmente, por tetracloreto de carbono $\left(\mathrm{CCl}_{4}\right)$ ou ligadura de ducto biliar, apresentam aumento do estresse oxidativo em tecido hepático ${ }^{(17,19,20)}$, mas a interrelação das alterações hepáticas e pulmonares ainda não está bem esclarecida.

O objetivo deste trabalho foi o de avaliar o estresse oxidativo e seu potencial antioxidante no tecido pulmonar de ratos com SHP induzida por ligadura de ducto biliar, bem como as alterações gasométricas e a relação pneumossomática.

\section{MATERIAIS E MÉTODOS}

Foram utilizados 12 ratos machos Wistar, com peso entre 200 e 300 g, divididos em dois grupos de 6 animais: um grupo cirrótico (Ci) e um controle (Co). A cirrose hepática foi produzida pela ligadura do ducto biliar e o grupo controle foi submetido a laparotomia e a manipulação do ducto biliar. Este modelo experimental seguiu o padrão descrito por KONTOURAS et al. ${ }^{(15)}$.

Os animais foram sacrificados após 28 dias do início do experimento.

Os procedimentos com os animais foram os preconizados pela Comissão de Pesquisa e Ética em Saúde do Grupo de Pesquisa e Pós-Graduação do Hospital de Clínicas de Porto Alegre, RS. ${ }^{(13)}$.

Para verificação da integridade hepática foram utilizadas as dosagens sangüíneas das enzimas aspartato aminotransferase (AST), alanino aminotranferase (ALT) e fosfatase alcalina (FA).

No homogeneizado de tecido pulmonar, realizou-se a avaliação do estresse oxidativo através dos métodos de substâncias reativas ao ácido tiobarbitúrico (TBA-RS) e quimiluminescência (QL). O TBA-RS é uma técnica em que se verifica a presença de malondialdeído e de outras substâncias, provenientes da peroxidação lipídica no material biológico, com resultados expressos em nanomol por miligrama de proteína $(\mu \mathrm{mol} / \mathrm{mg}$ prot $)^{(4)}$. A QL se baseia em reação iniciada por hidroperóxido de tert-butila, onde se avalia a emissão de elétrons livres produzidos por amostra, com resultados expressos em contagens por segundo por miligrama de proteína (cps $/ \mathrm{mg}$ prot) ${ }^{(14)}$. A avaliação da atividade antioxidante da enzima superóxido dismutase (SOD), que cataliza a dismutação do radical superóxido para formar peróxido de hidrogênio e oxigênio, foi verificada pelo sistema de detecção adrenalinaadrenocromo, e os resultados foram expressos em unidades de SOD por miligrama de proteína do homogeneizado do tecido em estudo ${ }^{(18)}$. Os testes de avaliação da integridade hepática foram realizados a partir da coleta de sangue do seio orbital dos ratos e as análises dos gases arteriais $\left(\mathrm{PaO}_{2}\right.$, pressão parcial de oxigênio arterial; $\mathrm{PaCO}_{2}$, pressão parcial de dióxido de carbono arterial; $\mathrm{SaO}_{2} / \mathrm{Hb}$, saturação de oxigênio da hemoglobina) foram realizadas através da coleta de sangue da aorta abdominal.

Os testes laboratoriais foram realizados no Laboratório Central da Irmandade Santa Casa de Misericórdia de Porto Alegre, RS, e os demais procedimentos foram realizados no Laboratório de Fisiologia Digestiva e Estresse Oxidativo do Instituto de Ciências Básicas da Universidade Federal do Rio Grande do Sul, Porto Alegre, RS..

Análise estatística realizada entre os grupos foi ANOVA, seguida de teste $t$ de Student. Todos resultados foram expressos como média \pm erro padrão. A diferença estatisticamente significativa foi definida quando $P<0,05$.

\section{RESULTADOS}

Os resultados estão apresentados em tabelas e gráficos nos diferentes grupos experimentais.

A Tabela 1 apresenta os valores das aminotransferases hepáticas e da FA, nas quais observou-se aumento significativo, bem como alterações significativas na gasometria arterial, na diferença artério-alveolar de oxigênio e na relação pneumossomática dos animais Co em relação aos animais Ci.

TABELA 1 - Valores de aminotransferases, fosfatase alcalina, gasometria arterial e relação pneumossomática dos animais Co e Ci

\begin{tabular}{ccc}
\hline Co & $\mathrm{Ci}$ \\
$\mathrm{n}=6$ & $\mathrm{n}=6$ \\
\hline
\end{tabular}

\begin{tabular}{lcc} 
Testes laboratoriais & \\
ALT (U/L) & $78,75 \pm 18,83$ & $162,75 \pm 17,70^{*}$ \\
AST (U/L) & $105,25 \pm 21,50$ & $500,5 \pm 45,15 * *$ \\
$\mathrm{FA}(\mathrm{U} / \mathrm{L})$ & $160 \pm 20,45$ & $373,25 \pm 45,44^{*}$ \\
& & \\
Gasometria arterial & & \\
$\mathrm{pH}$ & $7,34 \pm 0,0$ & $7,24 \pm 0,03 *$ \\
$\mathrm{PaO} 2(\mathrm{~mm} \mathrm{Hg})$ & $85,25 \pm 4,0$ & $49,9 \pm 11,3 *$ \\
$\mathrm{PaCO} 2(\mathrm{~mm} \mathrm{Hg})$ & $64,0 \pm 5,14 *$ & $9,8 \pm 3,3$ \\
$\mathrm{SaO} 2 / \mathrm{Hb}(\%)$ & $95,0 \pm 0,7$ & $73,3 \pm 12,07 *$ \\
$\mathrm{~A}-\mathrm{aDO} 2(\mathrm{~mm} \mathrm{Hg})$ & $4,05 \pm 0,56$ & $15,4 \pm 5,0 * *$ \\
& & $0,53 \pm 0,01 *$ \\
Relação pneumossomática & $0,38 \pm 0,02$ & \\
\hline * diferença significativa entre o grupo Ci e o grupo Co $(P<0,05)$ & \\
* * diferença significativa entre $\circ$ grupo $\mathrm{Ci} \bullet \circ$ grupo $\mathrm{C}(\mathrm{P}<0,01)$
\end{tabular}




\section{Lipoperoxidação no pulmão}

Na Figura 1 estão expressos os valores da lipoperoxidação no tecido pulmonar dos animais do grupo Co e Ci. Nota-se que os animais cirróticos apresentam aumento significativo tanto nos níveis de lipoperoxidação (LPO) avaliadas por TBA-RS e QL, quanto na atividade da SOD em relação ao grupo controle $(P<0,05)$.

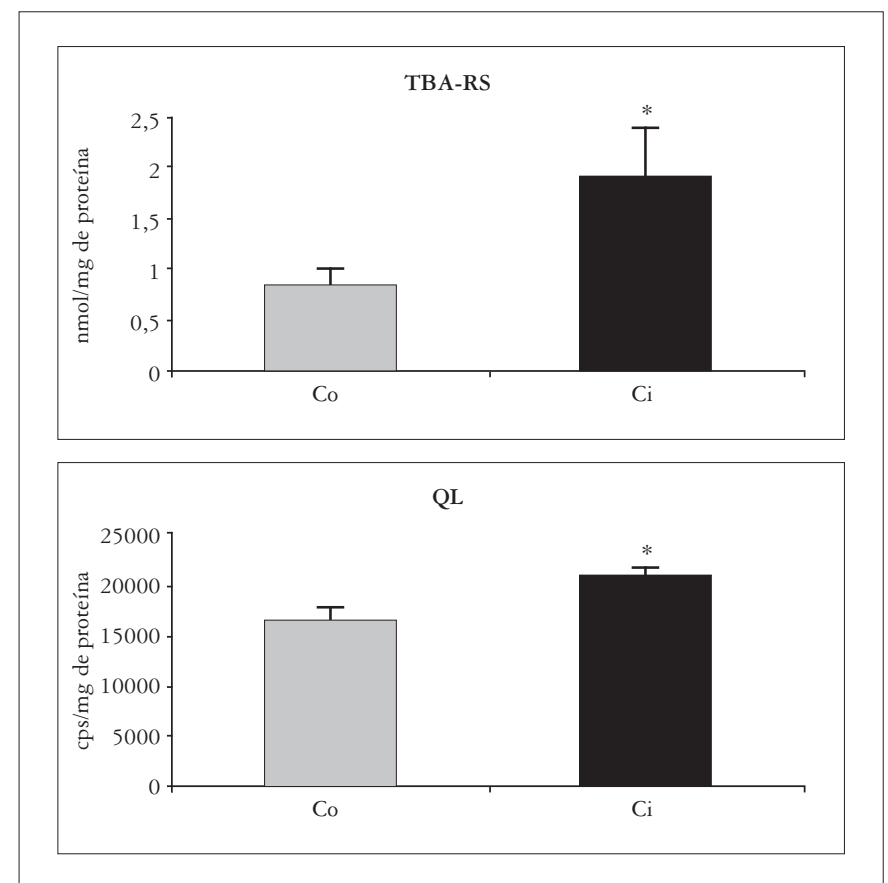

FIGURA 1 - Valores de lipoperoxidação por substâncias reativas ao acido tiobarbitúrico - TBA-RS e qumiluminescência - QL

* diferença significativa entre o grupo $\mathrm{Ci}$ e o grupo $\mathrm{Co}(P<0,05)$

\section{Enzima antioxidante no pulmão}

Houve aumento significativo da atividade da enzima antioxidante superóxido dismutase no tecido pulmonar dos animais cirróticos em relação aos animais controle $(P<0,05)$ (Figura 2).

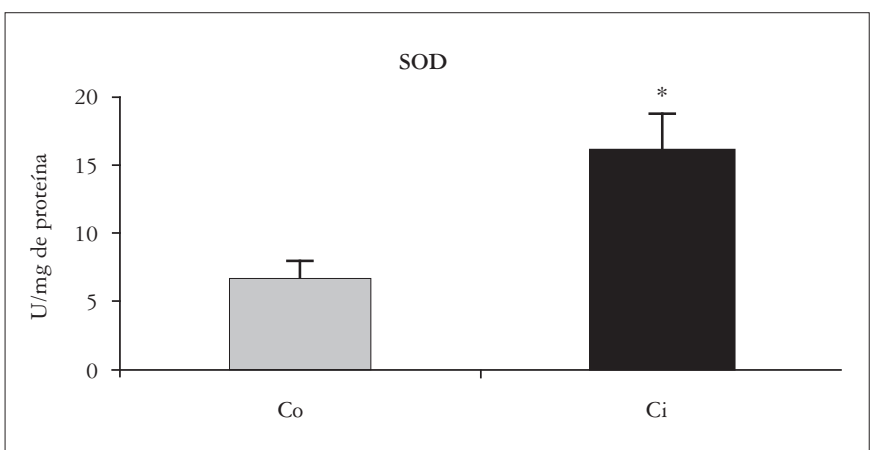

FIGURA 2 - Valores da atividade da enzima superóxido dismutase - SOD

Diferença significativa entre o grupo $\mathrm{Ci}$ e o grupo $\mathrm{Co}(P<0,05)$

\section{DISCUSSÃO}

A ligadura de ducto biliar é um dos modelos experimentais utilizados por diferentes grupos de investigação para produzir cirrose biliar secundária em animais. Este modelo de cirrose apresenta semelhanças em muitas das características observadas na cirrose em humanos e nas induzidas por $\mathrm{CCL}_{4}$, tais como a retenção de sódio, circulação hiperdinâmica, redução da resistência vascular periférica e aumento do débito cardíaco ${ }^{(12)}$

Segundo estudos realizados por KONTOURAS et al. ${ }^{(15)}$, 15 dias após a ligadura de ducto biliar (LDB) as alterações metabólicas e bioquímicas não se estabeleceram completamente. Desta maneira, no presente experimento, os animais foram sacrificados após 28 dias da LDB, onde em trabalhos de diversos pesquisadores já estão estebelecidas as condições de cirrose neste modelo.

Nas observações de PERES ${ }^{(20)}$ e MILTERSTAINER et al. ${ }^{(17)}$ ficaram bem estabelecidas as condições de cirrose em 28 dias de LDB, razão pela qual neste dia foram sacrificados os animais.

O aumento da FA, após a indução da colestase, dá-se pela elevação da síntese da enzima pelos hepatócitos. Os valores séricos encontrados após este período originam-se predominantemente do epitélio biliar $^{(23)}$.

Houve uma elevação das aminotransferases (ALT e AST), que pode ser atribuída à lesão ou destruição dos tecidos ricos em aminotransferases ou ainda a alterações da permeabilidade da membrana celular, que permitem sua liberação na corrente sanguínea ${ }^{(23)}$.

Neste estudo, os animais Ci tiveram os níveis séricos de AST, ALT e FA aumentados em relação ao grupo Co, comprovando a lesão de parênquima e da via biliar (Tabela 1).

Doenças crônicas do fígado, especialmente a cirrose, podem ser associadas com hipoxemia arterial, que resulta da combinação de alteração na relação ventilação-perfusão e shunt pela dilatação vascular intrapulmonar ${ }^{(1,7)}$.

Trabalhos experimentais têm demonstrado, no grupo Co, valores médios de $\mathrm{PaO}_{2}$ de $92,79 \mathrm{~mm} \mathrm{Hg}$ e de $\mathrm{PaCO}_{2}$ em torno de 40,01 $\mathrm{mm} \mathrm{Hg}$; em animais com 5 semanas de cirrose por LDB, os valores médios de $\mathrm{PaO}_{2}$ foram de $84,21 \mathrm{~mm} \mathrm{Hg}$ e de $34,94 \mathrm{~mm} \mathrm{Hg}$ para a $\mathrm{PaCO}_{2}{ }^{(11)}$.

Observou-se na presente série, que a $\mathrm{PaO}_{2}$ e a $\mathrm{SatO}_{2} / \mathrm{Hb}$ dos animais $\mathrm{Ci}$ tiveram diminuição significativa em relação ao grupo Co de, respectivamente $42 \%$ e $23 \%$, e que a $\mathrm{PaCO}_{2}$ apresentou aumento significativo em relação aos mesmos de $36 \%$, que pode ser devido às alterações que ocorrem nas trocas gasosas entre 0 tecido pulmonar e o sistema sanguíneo arterial (Tabela 1).

Em relação à diferença alvéolo-arterial, observou-se nesta casuística, diferença do grupo Ci em relação ao grupo Co superior à $100 \%$, o que também foi observado em estudo desenvolvido por LUO et al. ${ }^{(16)}$, onde os animais desenvolveram além de extensa diferença alvéolo-arterial, progressiva hiperventilação e hipoxemia. Estes resultados são similares aos encontrados em humanos.

BERTHELOT et al. ${ }^{(2)}$, encontraram aumento no diâmetro das arteríolas do tecido pulmonar em animais submetidos a LDB. 
No presente estudo, fez-se a relação do peso do pulmão com o peso corporal total (relação pneumossomática) (Tabela 1) e observou-se aumento do peso pulmonar no grupo $\mathrm{Ci}$ em relação ao grupo $\mathrm{Co}$, sendo que essas alterações podem ser devidas ao shunt e à dilatação vascular intrapulmonar. Possivelmente, essas alterações podem ter sido influenciadas pela congestão capilar intrapulmonar, fator este não avaliado neste estudo.

A vasodilatação intrapulmonar presente nesses casos, pode estar associada à translocação bacteriana, que ocorre devido à ausência ou diminuição de bile no intestino delgado, leva à perda do efeito emulsificante e antiendotóxico dos sais biliares, provocando níveis elevados de endotoxinas no intestino grosso, que serão absorvidas através da circulação portal, favorecendo a translocação bacteriana ${ }^{(6)}$. Esta tem sido encontrada em $45 \%$ $75 \%$ dos animais com cirrose experimental por $\mathrm{LDB}^{(21)}$.

A lesão hepatocelular impede a adequada filtração do sangue portal, promovendo o desenvolvimento de shunts portossistêmicos e dramática diminuição da capacidade fagocitária do fígado, permitindo a entrada de bactérias e endotoxinas na circulação pulmonar. Nessa situação, o pulmão filtra o sangue, compensando o decréscimo da função fagocitária do fígado ${ }^{(21)}$.

$\mathrm{O}$ aumento na atividade fagocitária pulmonar gera o acúmulo de macrófagos no endotélio pulmonar. Durante a fagocitose, a ativação numerosa de macrófagos secreta produtos para o meio extracelular, incluindo citocinas e óxido nítrico (NO).

$\mathrm{O} \mathrm{NO}$ age como um sinalizador molecular mediando a vasodilatação, quando produzido em baixas concentrações pela óxido nítrico sintase (NOS) em células vasculares endoteliais, e como fonte altamente tóxica oxidante, quando produzido em altas concentrações pela NOS em macrófagos no processo patológico ${ }^{(25)}$.

As células fagocitárias têm bem caracterizada a geração de ânion superóxido $\left(\mathrm{O}_{2}\right)$, que é capaz de oxidar a membrana plasmática, produzindo grande quantidade de espécies reativas do oxigênio (ERO) ${ }^{(25)}$.

No presente estudo, a avaliação da LPO no homogeneizado de pulmão de ratos Co e Ci pós-LDB demonstrou, pelas técnicas de TBA-RS e QL, aumento significativo da peroxidação lipídica, possivelmente devido à ação de células fagocitárias no combate ao processo de translocação bacteriana (Figura 1).

A migração de macrófagos para o pulmão leva à produção de radicais superóxido, o que requer aumento da atividade enzimática antioxidante, vista no presente trabalho, através do aumento significativo da atividade da SOD no homogeneizado de tecido pulmonar no grupo cirrótico na tentativa de proteger o órgão da agressão dos radicais livres aí formados (Figura 2).

Parece que o modelo de cirrose induzida por LDB contribui para o estudo da SHP, visto que se observaram alterações da gasometria arterial, aumento da lipoperoxidação e das defesas antioxidantes aqui representadas pela enzima SOD.

\section{CONCLUSÕES}

Os dados obtidos sugerem que o modelo experimental da LDB apresentou as principais características da SHP e sugere-se que o mesmo possa ser utilizado para futuros estudos envolvendo estresse oxidativo nas alterações hepáticas e a sua relação com o sistema respiratório.

Tieppo J, Vercelino R, Dias AS, Marroni CA, Marroni N. Common bile duct ligation as a model of hepatopulmonary syndrome and oxidative stress. Arq Gastroenterol 2005;42(4):244-8.

ABSTRACT - Background - The hepatopulmonary syndrome is characterized by hepatic dysfunction and presence of dilated pulmonary vessels, with alterations in air diffusion that can be demonstrated in the experimental model of common bile duct ligation. Aim - To evaluate the oxidative stress in pulmonary tissue of cirrhotic rats with common bile duct ligation. Material/Methods- We used 12 male Wistar rats weighing between 200-300 g divided in two groups: control $(\mathrm{Co}=6)$ and cirrhotic $(\mathrm{Ci}=6)$. We evaluated aminotransferases, arterial gasometry, lipoperoxidation and chemoluminescence), and antioxidant enzymatic activity with superoxide dismutase. The tissues analyzed for hepatopulmonary syndrome were cirrhotic liver and lung. Results- The animals with common bile duct ligation showed alterations in the following aminotransferases: aspartate aminotransferase, $\mathrm{Co}=105.3 \pm 43 / \mathrm{Ci}=500.5 \pm 90.3$, alanine aminotransferase, $\mathrm{Co}=78.75 \pm$ $37.7 / \mathrm{Ci}=162.75 \pm 35.4$, and alkaline phosphatase, $\mathrm{Co}=160 \pm 20.45 / \mathrm{Ci}=373 \pm 45.44$. The lipoperoxidation and the antioxidant response had significant differences between the groups when evaluated in lung (lipoperoxidation) $\mathrm{Co}=0.87 \pm 0.3 / \mathrm{Ci}=2.01 \pm 0.9$, chemoluminescence Co $=16008.41 \pm 1171.45 / \mathrm{Ci}=20250.36 \pm 827.82$ superoxide dismutase $\mathrm{Co}=6.66 \pm 1.34 / \mathrm{Ci}=16.06 \pm 2.67$. Conclusions - Our results suggest that in this experimental model of cirrhosis using common bile duct ligation, there is an increase in lipoperoxidation in pulmonary tissue as well as an increase in superoxide dismutase's antioxidant activity, suggesting a pulmonary injury caused by secondary biliary cirrhosis.

HEADINGS - Oxidative stress. Common bile duct ligation. Hepatopulmonary syndrome. 


\section{REFERÊNCIAS BIBLIOGRÁFICAS}

1. Andersen BL, Gordon L, Buse MG. Intrapulmonary shunting associated with cirrhosis: incidental diagnosis by perfusion lung scan. Clin Nucl Med 1982;7:108-10.

2. Berthelot P, Walker PG, Sherlock S, Reid L. Arterial changes in the lungs in cirrhosis of ihe liver-lung spider nevi. N Engl J Med 1966;274:291-8.

3. Brandão ABM, Marroni CA. Testes de função hepática. In: Mattos AA, Dantas W, editores. Compêndio de hepatologia. 2.ed. São Paulo: BYK Fundo; 2001

4. Buege JA, Aust SD. Microssomal lipid peroxidation. Meth Enzimol 1978;52:302-9.

5. Chang SW, Ohara N. The lung in liver disease. Clin Chest Med 1996;17:1-17.

6. Cruz CAT, Figueiredo F, Beer Jr A, Silva Jr OC. Alterações imunológicas na obstrução biliar extra-hepática. In: Silva OC, Zucoloto S, Beer A, editores. Modelos experimentais de pesquisa em cirurgia. São Paulo: Robe; 1998.

7. Dal Palu C, Donaggio G, Dal Zotto I, Pessina AC. Arteriovenous shunts in cirrhotic patients studied with human serum albumin macroagragates tagged with I-131 (MAA131-I). Scand J Gastroenterol 1968;3:425-31.

8. Garcia E, Silverio A, Cassal A. Síndrome hepatopulmonar: fisiopatogenia e opções terapêuticas. GED Gastroenterol Endosc Dig 1998;17:53-62.

9. Garcia E, Silvério AO, Malmann M, Pereira-Lima JE, Moreira JS. Síndrome hepatopulmonar. J Pneumol 1996;22:279-84.

10. Fallon MB, Abrams GA, Luo B, Hou Z, Dai J, Ku DD. The role of endothelial nitric oxide synthase in the pathogenesis of a rat model of hepatopulmonary syndrome. Gastroenterology 1997;113:606-14.

11. Fallon MB, Abrams GA, McGrath JW, Hou Z, Luo B. Common bile duct ligation in the rat: a model of intrapulmonary vasodilatation and hepatopulmonary syndrome. Am J Physiol 1997;272:G779-G84.

12. Fernandes AO, Mies S. Hipertensão portal. In: Silva OC, Zucoloto S, Beer A. modelos experimentais de pesquisa em cirurgia. São Paulo: Robe; 1998.

13. Goldin JR, Raymundo MM. Pesquisa em saúde e direito dos animais. 2.ed. Porto Alegre: HCPA; 1997.

14. Gonzalez-Flexa B, Llesuy S, Boveris A. Hydroperoxide-initiated chemiluminescence: on assay for oxydative stress in biopsies of liver, heart, and muscle. Free Rad Biol Med 1991;10:41-7.
15. Kountouras J, Billing BH, Scheuer PJ. Prolonged bile duct obstruction: a new experimental model for cirrhoses in the rat. Br J Exp Pathol 1984;65:305-11.

16. Luo B, Abrams GA, Fallon MB. Endothelin-1 int the rat bile duct ligation model of hepatopulmonary syndrome: correlation with pulmonary dysfunction. J Hepatol 1998;29:571-8.

17. Miltersteiner A, Milterstainer D, Pereira-Filho N, Frota AR, Ely PB, Zettler CG, Marroni CA, Marroni NP. Uso da quercetina a longo prazo em ratos cirróticos. Acta Cir Bras 2003; 18:232-7.

18. Misra HP, Fridovich I. The role of superoxide anion in the autoxidation of epinephrine and a simple assay for superoxide dismutase. J Biol Chem 1972;247:3170-5.

19. Pavanato MA, Tunón MJ, Sánchez-Campos S, Marroni CA, Lessuy S, González-Gallego J, Marroni NP. Effects of quercetin on liver damage in rats with carbon tetrachlorideinduced cirrhosis. Dig Dis Sci 2003;48:824-9.

20. Peres W. Radicales libres y ciroosis biliar secundaria: efecto protector de los flavonoides quercitina e rutina [tese]. León: Universidad de León; 1999.

21. Rabiller A, Nunes H, Lebrec D, Tazi KA, Wartski M, Dulmet E, Libert JM, Mougeot C, Moreau R, Mazmanian M, Humbert M, Hervé P. Prevention of gram-negative translocation reduces the severity of hepatopulmonary syndrome. Am J Respir Crit Care Med 2002;166:514-7.

22. Rhoden EL, Mauri M, Petteffi L, Klein AB, Rhoden CR. O estresse oxidativo na cirrrose hepática induzida por tetracloreto de carbono em ratos. GED Gastroenterol Endosc Dig 1997;16:47-50.

23. Santos JS, Zucoloto S, Silva Jr OC, Salgado Jr W, Ceneviva R. Cirrose biliar secundária. In: Silva OC, Zucoloto S, Beer A, editores. Modelos experimentais de pesquisa em cirurgia. São Paulo: Robe; 1998.

24. Silva Jr OC, Mazzetto SA, Souza MEJ, Picinato MANC, Sankarankutty AK. Obstrução biliar extra hepática. In: Silva OC, Zucoloto S, Beer A, editores. Modelos experimentais de pesquisa em cirurgia. São Paulo: Robe; 1998.

25. Thannickal VJ, Fanburg BL. Reactive oxygen species in cell signaling. Am J Physiol Lung Cell Mol Physiol 2000;279:L1005-L28. 